\title{
Editorial 2009
}

\section{Francesco Battaglia}

Statistical Methods and Applications is attracting an increasingly large interest. Our Journal has been accepted for Science Citation Index Expanded and will appear for the first time in 2009 Journal of Citation Reports, published in 2010.

We receive a large number of good quality submissions and tried to increase the number of printed pages. While last year the number of issues per year was increased from 3 to 4, this year also we have been able to ensure a further slight increase, and from 2009 each issue will be about 150 pages for a total of 600 pages each volume.

Statistical Methods and Applications has started electronic management. On adopting the Editorial Manager platform, all stages of the evaluation and production process are performed online, providing better timeliness and safety. Moreover, the technical check calls for a more precise preparation of the manuscript, and this provides a better overall quality, though requiring the authors for a little bit more attention.

The editorial board makes the best effort to keep reviewing time as short as possible, in spite of the large number of submissions. The average time to first answer was about 75 days in 2008, though the variance is still larger than desired. Papers are evaluated, together with an associate editor, at least by two referees. The acceptance rate in 2008 was about $26 \%$.

The editorial board has several new members, since most associate editors terminated their four-year appointment: M. M. Barbieri, A. Decarli, I. Drudi, G. Lovison, G. Montanari, T. Proietti, C. Rampichini, S. Salvini, M. Vichi. The Journal acknowledges their invaluable action and thanks them warmly. The associate editors V. Buratta. M. Gasparini, A. Giovagnoli, B. Liseo, P. Rigo and N. Tedesco were asked to keep their role for one more year, and all agreed. And here are the new appointed editors.

\footnotetext{
F. Battaglia $(\varangle)$

Dipartimento di Statistica, Probabilità e Statistiche Applicate,

Sapienza University of Rome, 00100 Rome, Italy

e-mail: francesco.battaglia@uniroma1.it
} 
For the Methods Section Lucio Barabesi, Roberto Baragona, Francesco Bartolucci, Anna Clara Monti, Roberto Rocci; for the Applications Section Pierluigi Daddi, Alessandra De Rose, Paolo Giudici, Fabrizia Mealli. As the Editor of Statistical Methods and Applications, it is a pleasure to welcome the new associate editors, with their help we shall try to offer our audience an even more interesting and useful Journal. 